\title{
ALGEBRAS OF HOLOMORPHIC FUNCTIONS ON ONE-DIMENSIONAL VARIETIES
}

\author{
BY \\ HUGO ROSSI( ${ }^{(1)}$
}

1. Introduction. By $C^{n}$ we mean $n$-dimensional complex vector space. For $z \in C^{n}$, we will represent the ring of germs of holomorphic functions at $z$ by $\Theta_{z}^{n}$, and the sheaf of germs of holomorphic functions in $C^{n}$ by $\Theta^{n}$ (for sheaftheoretic terminology, see $[2 ; 5])$. In general, a ringed space is a pair $(X, \Theta)$, where $X$ is a locally compact Hausdorff space and $\Theta$ is a sheaf of rings of germs of continuous functions on $X$ (see [6]). Thus $\left(C^{n}, \Theta^{n}\right)$ is a ringed space. By holomorphic function on the ringed space $(X, \Theta)$ we mean a continuous function on $X$ whose germ at any $x \in X$ is in $\Theta_{x}$. If $U$ is an open subset of $X,(U, \Theta \mid U)$ is a ringed space; we will let $H(U, \Theta)$ denote the ring of holomorphic functions on $U$. Where it is clear what $\Theta$ is we will write $H(U)$ for $H(U, \Theta)$.

Let $U$ be an open set in $C^{n}$, and $f_{1}, \cdots, f_{t} \in H\left(U, \Theta^{n}\right)$. Let $V=\left\{z \in U ; f_{i}(z)=0,1 \leqq i \leqq t\right\}$. We will write $V=V\left(f_{1}, \cdots, f_{\imath}\right)$. We consider $\Theta^{n}$ as defining a sheaf of germs of continuous functions on $V$, and write $\Theta^{V}=\Theta^{n} \mid V$. Then $\left(V, \Theta^{V}\right)$ is a ringed space. A function $f$ defined on $V$ is thus holomorphic on $V$ if, for every $x \in V$, there is a neighborhood $W$ of $x$ in $C^{n}$ such that $f \mid W \cap V$ is the restriction to $W \cap V$ of a function holomorphic in $W$. Any closed subset of a domain $U$ in $C^{n}$ which is locally given as the zeros of a finite number of holomorphic functions is called a variety.

Let $(X, \Theta),(Y, \Phi)$ be ringed spaces and $\phi: X \rightarrow Y$ a continuous map. By $\hat{\phi}(\Phi)$ we mean the sheaf on $X$ whose stalk at $x \in X$ is the set $\left\{f \circ \phi ; f \in \Phi_{\phi(x)}\right\}$. If $\hat{\phi}(\Phi) \subset \Theta$, we say $\phi$ is holomorphic. If $\phi$ is a homeomorphism and $\phi^{-1}$ is also holomorphic, we say $\phi$ is biholomorphic.

A complex manifold is a ringed space $(X, \Theta)$ with the following property: for every $x \in X$, there is a neighborhood $U$ of $x$ and a biholomorphic map of $(U, \Theta)$ onto $\left(W, \Theta^{n}\right)$, where $W$ is an open set in $C^{n}$. An analytic space is a ringed space $(X, \Theta)$ with the following property: for every $x \in X$, there is a neighborhood $U$ of $x$ and a biholomorphic map of $(U, \Theta)$ onto $\left(V, \Theta^{v}\right)$, where $\left(V, \Theta^{v}\right)$ is a variety.

Let $(S, \Theta)$ be an analytic space. A point $x \in S$ is regular if $x$ has a neighbor-

Received by the editors January 9, 1960 and, in revised form, January 12, 1961.

(1) Most of this work was done while the author was a General Electric Fellow at the Massachusetts Institute of Technology. This research was partially supported by the United States Air Force through the Air Force Office of Scientific Research of the Air Research and Development Command under Contract Number AF 49(638)692. Reproduction in whole or in part is permitted for any purpose of the United States Government. 
hood $U$ such that $(U, \Theta)$ is a complex manifold. The set $S_{\text {rez }}$ of regular points of $S$ forms a complex manifold dense in $S$. By a one-dimensional analytic space we mean an analytic space $S$ such that $S_{\text {reg }}$ is a one-dimensional manifold. In this case $S_{\text {sing }}=S-S_{\text {reg }}$ is a discrete set of points on $S$ (see [2]).

If $K$ is a compact subset of an analytic space, let $H(K)$ be the ring of all functions holomorphic in a neighborhood of $K$. Let $A(K)$ be the uniform closure on $K$ of $H(K) . A(K)$ is a Banach algebra with the uniform norm. Our main purpose is to study $A(K)$ with respect to the property of being a maximal subalgebra of $C(\Gamma(A(K)))$, the algebra of all continuous functions on the Silov boundary of $A(K)$. This will be done under the restriction that $K$ consists of a domain bounded by finitely many piecewise analytic curves. It has been shown, in the case where $S$ is a Riemann surface, that $A(K)$ is maximal in $C(\Gamma(A(K))$ ) (Wermer, Royden $[9 ; 10]$ ). This result is our starting point. In particular, we will associate with any one-dimensional analytic space $S$ a one-dimensional complex manifold $R$, and a projection $\pi: R \rightarrow S$, and with compact $K$ on $S$, a compact $\tilde{K}$ on $R$. We shall show that, in a natural sence, $A(K)$ is a subalgebra of $A(\tilde{K})$ of finite codimension in $A(\tilde{K})$; this fact is the basis of the discussion.

We shall also attempt to describe certain subalgebras of $A(K)$, for $K$ a compact subset of a Riemann surface. These subalgebras are of the form $A_{0}(K, U)$, the uniform closure on $K$ of a subalgebra $A_{0}$ of $H(U)$, where $U$ is a neighborhood of $K$. The first result in this direction is Wermer's theorem.

1.1. Theorem (Wermer [11]). Let $R$ be a Riemann surface, $\Gamma$ a simple closed analytic curve on $R$ such that $\Gamma$ is the boundary of a domain $D$ with $D \cup \Gamma$ compact. Let $f, g$ be holomorphic in a neighborhood of $D \cup \Gamma, d f$ does not vanish on $\Gamma$ and $f, g$ together separate points on $\Gamma$. Then there is a finite subset $T$ of $D \cup \Gamma$ and an integer $n$ such that if $h$ is in $A(D \cup \Gamma)$, and $h$ vanishes at each point of $T$ with order no less than $n$, then $h$ is approximable on $D \cup \Gamma$ by functions in the algebra $\{f, g\}$ of polynomials in $f$ and $g$.

In the appendix of [11] he extends this to finitely many functions. The crucial feature of this theorem is to prove that $D \cup \Gamma$ is $\{f, g\}$-convex (see the definition below); with this assumption the proof depends only on theorems of several complex variables of a general nature.

Later Bishop proved a closely related, but essentially different theorem.

Definition. Let $U$ be an open set on an analytic space $S$, and $K$ a compact set in $U$. Let $A_{0}$ be a subalgebra of $H(U)$. We say $K$ is $A_{0}$-convex if $K=\left\{x \in U ;|f(x)| \leqq \sup \{|f(y)| ; y \in K\}\right.$ for all $\left.f \in A_{0}\right\}$. For $V \subset U$ open, we say $V$ is $A_{0}$-convex if $V$ can be written as the increasing union of a sequence of $A_{0}$-convex compact sets.

1.2. Theorem (Bishop [3]). Let $R$ be an open Riemann surface, $K$ a compact subset of $R, B$ a subalgebra of $H(R)$ such that (i) $K$ is $B$-convex, (ii) $\{p \in R$; 
there is $a q \neq p$ in $R$ such that $f(p)=f(q)$ for all $f \in B\} \cup\{p \in R ; d f(p)=0$ for all $f \in B\}$ (the singular set relative to $B$, denoted by $S(B)$ ) is finite.

Then there is a positive integer $N$ such that $\bar{B}$, the closure of $B$ on $K$, contains the ideal of $C(K) \cap H$ (int $K$ ) consisting of those functions which vanish on $S(B) \cap K$, and vanish of order at least $N$ at those points of $S(B)$ interior to $K$.

The main point here is the approximation theorem, which we shall use in the maximality discussion. In $\S 6$ we shall prove the following theorem.

6.7. THEOREM. Let $K$ be a compact subset of a Riemann surface $R$, and let $U$ be a neighborhood of $K$. Suppose $A^{0}$ is a subalgebra of $H(U)$ such that (i) $A^{0}$ is generated by $f_{1}, \cdots, f_{n} \in H(U)$, (ii) $K$ is $A^{0}$-convex, (iii) $\{p \in U$; there is a $q \in U$ such that $p \neq q$ but $f(p)=f(q)$ for all $\left.f \in A^{0}\right\}$ is finite. Let $A$ be the uniform closure of $A^{0}$ on $K$.

Then there is a variety $V$ in the unit polycylinder of some $C^{t}$ and a map $\phi$ of a neighborhood of $K$ onto $V$ such that

$$
A=\{f \circ \phi ; f \text { in the closure of the polynomials on } \phi(K)\} .
$$

Together these three theorems give a complete description of $A_{0}(K, U)$ when it is finitely generated.

This work was originally done at the Massachusetts Institute of Technology as part of my doctoral thesis. I am greatly indebted to my teachers, Professors Singer and Hoffman, and to the department of mathematics at Massachusetts Institute of Technology for all the assistance I received.

2. Ideals of $A(K)$ on a Riemann surface. Let $R$ be a compact Riemann surface, and $S$ any subset of $R$. We write $M(S)$ for the vector space of functions, meromorphic on $R$ which are regular on $S$. From the Riemann-Roch theorem we obtain the following result.

2.1. Lemma. Let $U$ be any open set on $R$.

(i) Given $p \neq q$ in $U$, there is an $f \in M(U)$ such that $f(p) \neq f(q)$.

(ii) Given $p, p_{1}, \cdots, p_{t}$ in $U$, and positive integers $n, n_{1}, \cdots, n_{t}$, there is an $f \in M(U)$ with zeros of orders $\geqq n_{i}$ at $p_{i}, 1 \leqq i \leqq t$ and a zero of order $n$ at $p$ (i.e., if $(U, z)$ is a local parameter at $p, f$ has the Taylor series in $U: f=\sum a_{n} z^{n}$ with $a_{i}=0, i<n$, and $a_{n} \neq 0$ ).

Proof. (i) Let $p \neq q$ in $U$; by the Riemann-Roch theorem, there is a meromorphic function $f$ regular on $U$ with a zero of some order $k$ at $p$. If $f(q) \neq 0$, then $f(p) \neq f(q)$. Suppose $f$ has a zero of order $m$ at $q$. Let $g$ be meromorphic on $R$, with a pole only at $q$ of order, say, $n$. Then $f^{n} g^{k}(q) \neq 0$, but $f^{n} g^{k}(p)=0$, so $f^{n} g^{k}$ is the required function.

(ii) Let now $f \in M(U)$ such that $f$ has a zero of order at least $n_{i}$ at $p_{i}$, and is zero at $p$. Let $k$ be the order of the zero at $p$. Again by the RiemannRoch theorem there is a meromorphic function $g$ whose only pole is at $p$ and is of order $m$, where $m$ is prime to $p$, so long as $m$ is large enough. Then 
there are positive $s, t$ such that $s k-t m=1$. Then $f^{s} g^{t}$ is in $M(U)$ and has a zero of order 1 at $p$. Thus $\left(f^{s} g^{t}\right)^{n}$ has a zero of order $n$ at $p$, and obviously still has zeros of orders at least $n_{i}$ at $p_{i}$.

Now let $K$ be a compact proper subset of $R . M(K)$ is contained in $H(K)$, so the closure $A(K)$ of $H(K)$ in the uniform norm on $K$ contains the restriction to $K$ of functions in $M(K)$. We use this fact to show that the structure space $S(A(K)$ ) of $A(K)$, (the set of complex holomorphisms of $A(K)$ ) is just $K$.

\subsection{LEMMA. $S(A(K))=K$.}

Proof. By Lemma 2.1, $M$ separates the points of $K$; thus $A(K)$ separates points of $K$. Every point of $K$ thus determines a continuous homomorphism of $A(K)$, so $S(A(K))$ contains $K$.

Let $U$ be a neighborhood of $K$. Let $h$ be in $S(A(K))$, different from evaluation at some point $r$ in $K$. Let $F$ be meromorphic on $R$ with its only pole in $U$ a simple pole at $r$. Now there is a $g$ in $H(K)$ such that $h(g)=1$, $g(r)=0$. Then $g F$ is in $H(K)$. Let $t=h(g F)$; then for $P=F-t, g P$ is in $M(K)$ and $h(g P)=h(g(F-t))=h(g F)-t h(g)=0$. Let $r_{1}, \cdots, r_{n}$ be the zeros of $P$ on $K$ of order $m_{1}, \cdots, m_{n}$, respectively. If $h$ is not evaluation at any $r_{j}$, there are $f_{j}$ in $H(K), h\left(f_{j}\right)=1, f_{j}\left(r_{j}\right)=0$. Let $f=\left(\prod f_{j}^{m_{j}}\right) P^{-1} . f$ is in $H(K)$ and, since $f(r)=0, f P$ is also in $H(K)$. Thus $h\left(\prod f_{j}^{m_{j}}\right)=\prod h\left(f_{j}\right)^{m_{j}}=1$; but $\prod f_{j}^{m_{j}}=f P$, so $h\left(\prod f_{j}^{m_{j}}\right)=h(f P)=h(f P) h(g)=h(f P g)=h(f) h(g P)=0$, a contradiction. Thus $h$ is evaluation at $r$ or some $r_{j}$, i.e., evaluation at some point of $K$.

This proof is essentially a part of Arens' proof that the algebra $C(K)$ $\cap H($ int $K$ ) has $K$ as its structure space [1].

We now exploit the infinite dimensionality of $M(K)$ as a vector space to obtain a theorem which will be useful in the discussion of holomorphic functions on one-dimensional analytic spaces.

2.3. Theorem. Let $x_{1}, \cdots, x_{n} \in K$. Let $\left(U_{i}, z_{i}\right)$ be a local coordinate at $x_{i}$, and let $P_{i}(X)$ be polynomials of degree $n_{i}$ over the complex numbers. Then there is a $g \in M(K)$ such that the first $n_{i}$ terms of the Taylor expansion of $g$ about $x_{i}$ in $z_{i}$ agrees with $P_{i}\left(z_{i}\right)$.

Proof. For $f \in M(K)$, we can expand $f$ in a Taylor series in $z_{i}$ about $x_{i}: f=\sum \lambda_{i j}(f) z^{j} . \lambda_{i j}(f)$ is a linear functional on $M(K)$. According to 2.1 (ii) we can find an $f_{i j} \in M(K)$ such that $f_{i j}$ has a zero of order $j$ at $x_{i}$ and a zero of order at least $n_{r}$ at $x_{r}$. That is, $\lambda_{r s}\left(f_{i j}\right)=0$ if $r \neq i$ and $s \leqq n_{r}$, or if $r=i$ and $s<j$. Thus the matrix $M=\left(\lambda_{r s}\left(f_{i j}\right)\right), 1 \leqq r, i \leqq n, 0 \leqq s \leqq n_{r}, 0 \leqq j \leqq n_{i}$, is a $\left(\sum n_{i}\right)$ $\times\left(\sum n_{i}\right)$ triangular matrix all of whose diagonal entries are nonzero. Thus $M$ is nonsingular.

Write $P_{i}(X)=\sum a_{i j} X^{j}$. Let $a$ be the vector $\left(a_{i j}\right)$. Then there is a vector $b=\left(b_{i j}\right)$ such that $M b=a$. Let $g=\sum b_{i j} f_{i j}$; then $g \in M(K)$, and $\lambda_{r s}(g)$ $=\sum b_{i j} \lambda_{r s}\left(f_{i j}\right)=a_{r s} ;$ thus $g$ is the required function. 
2.4. Corollary. Let $x_{1}, \cdots, x_{n}$ be in the interior of $K$. Let $I=\{f \in A(K)$; $f$ has a zero of order $\geqq n_{i}$ at $\left.x_{i}\right\}$. Then $A(K)=M(K)+I$.

Proof. Let $\left(U_{i}, z_{i}\right)$ be a local parameter at $x_{i}$, and let $f \in A(K)$. By 2.3, there is a $g \in M(K)$ such that the Taylor expansion of $g$ in $z_{i}$ at $x_{i}$ coincides with that of $f$ up to the $n_{i}$ th term. Then $f-g \in A(K)$, and vanishes of order $\geqq n_{i}$ at $x_{i}$, so $f-g$ is in $I$. Thus $f=g+(f-g)$ is in $M(K)+I$.

3. Normalization of an analytic space. Let $S$ be a one-dimensional analytic space. We shall show that there is a complex manifold $R$ and a projection $\pi: R \rightarrow S$ such that for $U$ an open set on $S, \bar{U}$ compact, the set $\{f \circ \pi$; $f \in H(U)\}$ is of finite codimension in $H\left(\pi^{-1}(U)\right) . R$ will be the normal model for $S$ (see [6]).

Definition. Let $S$ be an analytic space. The normalization of $S$ is a pair $(R, \pi)$ such that: (i) $R$ is a locally compact, locally connected Hausdorff space; (ii) $\pi: R \rightarrow S$ is continuous, proper and onto; (iii) $\pi^{-1}(s)$ is finite for all $s$ in $S$ (in fact, $\pi^{-1}(s)=\left\{p_{1}, \cdots, p_{k(s)}\right\}$, where $k(s)$ is the number of irreducible components of $S$ at $x$ ); (iv) $\pi^{-1}\left(S_{\text {sing }}\right)$ separates no connected set in $R$ and is nowhere dense; (v) $\pi: R-\pi^{-1}\left(S_{\text {sing }}\right) \leftrightarrow S-S_{\text {sing }}$ is a homeomorphism.

The points of $R$ are pairs $(s, V), s \in S$ and $V$ an irreducible branch of $S$ at $s$, and $\pi(s, V)=s$. The basis neighborhoods are of the form $O(U, V)$ $=\pi^{-1}(U \cap V)$ where $V$ is an irreducible branch of $S$ in the open set $U$. The space $R$ is called the normal model of $S$.

Let $U \subset R$ be open. If $f: U \rightarrow C$ is continuous and $f \circ \pi^{-1} \mid U-\pi^{-1}\left(S_{\text {sing }}\right)$ is holomorphic, we say that $f$ is holomorphic on $U$. Let $\Theta^{R}$ be the sheaf of germs of holomorphic functions on $R$. Then $\pi$ is a homomorphic map.

The following facts were proven by Oka $[8 ; 6]$.

3.1. THEOREM. The normal model exists, is an analytic space and is unique up to a biholomorphic map with respect to the properties (i)-(v) and that $\pi$ is holomorphic.

3.2. ThEOREM. If $s \in S$ is an irreducible point, $\pi^{-1}(s)=p$. $\Theta_{p}^{R}$ is the integral closure of $\pi\left(\Theta_{s}^{S}\right)=\left\{f \circ \pi ; f \in \Theta_{s}^{S}\right\}$. There is a nonzero ideal $C$ of $\Theta_{p}^{R}$ such that $C \Theta_{p}^{R} \subset \pi\left(\Theta_{s}^{S}\right)$.

We now prove

3.3. TheOREM. The normal model of a one-dimensional analytic space is a one-dimensional complex manifold.

Proof. We know $R-\pi^{-1}\left(S_{\text {sing }}\right)$ is a complex manifold, since it is biholomorphic to $S-S_{\text {sing. }}$. Let $s \in S_{\text {sing. }}$. Since $S_{\text {sing }}$ is discrete, $s$ has a neighborhood $U$ such that $U \cap S_{\text {sing }}=\{s\}$. Let $V_{1}, \cdots, V_{t}$ be the branches of $S$ in $U$; then $\pi^{-1}(U)=\mathrm{U} O\left(U, V_{i}\right)$ and each $O\left(U, V_{i}\right)$ is connected. It is easy to see that $\pi: O\left(U, V_{i}\right) \leftrightarrow U \cap V_{i}$ is a homeomorphism and $\pi: O\left(U, V_{i}\right)-\left\{p_{i}\right\} \leftrightarrow U \cap V_{i}$ $-\{s\}$ is biholomorphic, where $p_{i}=\left(s, V_{i}\right)$. We need only prove that we can 
define a local coordinate at $p_{i}$ which, with the natural structure of $O\left(U, V_{i}\right)$ $-\left\{p_{i}\right\}$, makes of $O\left(U, V_{i}\right)$ a Riemann surface such that $\pi$ is still holomorphic. Then, by the uniqueness, this structure is the normalization structure. Thus, since we are interested in only $O\left(U, V_{i}\right)$ near $p_{i}$, we can assume $S$ has only one branch at $s$, further, that $S$ is embedded as a variety $V$ in a neighborhood of the origin $(s=0)$ in $C^{n}$.

3.5. Lemma. Let $V$ be an irreducible one-dimensional variety in $C^{n}$ at 0 , and suppose that 0 is the only singular point of $V$. Then there are a Riemann surface $R^{1}$ and a holomorphic homeomorphism $\rho: R^{1} \leftrightarrow V$ such that $\rho \mid R^{1}-\rho^{-1}(0)$ is biholomorphic.

Proof. In a neighborhood of 0 , by the Nullstellensatz [5, Chapter XIV] for a suitable choice of coordinates we can write $V-\{0\}=V\left(f_{1}, \cdots, f_{n-1}\right)$ where

$$
\begin{aligned}
& f_{1}\left(z_{1}, \cdots, z_{n}\right)=g_{1}\left(z_{1}, z_{2}\right)=z_{2}^{r}+\sum_{i=0}^{r-1} a_{i}\left(z_{1}\right) z_{2}^{i}, \\
& f_{j}\left(z_{1}, \cdots, z_{n}\right)=z_{j}-\frac{g_{j}\left(z_{1}, z_{2}\right)}{D\left(z_{1}\right)}
\end{aligned}
$$

where $g_{j}$ are distinguished polynomials, and $D(0)=0$.

Let $R^{1}$ be the Riemann surface covering $\left\{\left|z_{1}\right|<\epsilon\right\}$ of the algebraic function defined by $g_{1}\left(z_{1}, z_{2}\right)=0$, and $\pi^{0}: R^{1} \rightarrow\left\{\left|z_{1}\right|<\epsilon\right\}$ the projection. Let $x$ in $R^{1}, \pi^{0} x \neq 0$; then $x$ corresponds to a certain branch $\phi_{x}$ of this algebraic function. We define $\rho: R^{1} \rightarrow V \cap\left\{\left|z_{1}\right|<\epsilon\right\}:$

$$
\begin{aligned}
& \rho(x)=\left(\pi^{0} x, \phi_{x}\left(\pi^{0} x\right), \frac{g_{2}\left(\pi^{0} x, \phi_{x}\left(\pi^{0} x\right)\right)}{D\left(\pi^{0} x\right)}, \ldots, \frac{g_{n}\left(\pi^{0} x, \phi_{x}\left(\pi^{0} x\right)\right)}{D\left(\pi^{0} x\right)}\right), \\
& \rho(x)=0 \quad \text { if } \quad \pi^{0} x=0 .
\end{aligned}
$$

If $\pi^{1}$ is the projection of $V$ onto $\left\{\left|z_{1}\right|<\epsilon\right\}$ it is obvious that $\pi^{0}=\pi^{1} \circ \rho$.

$\rho$ is one-one. For if $\rho x=\rho y$, then $\pi^{0} x=\pi^{0} y, \phi_{x}\left(\pi^{0} x\right)=\phi_{y}\left(\pi^{0} y\right)=\phi_{y}\left(\pi^{0} x\right)$ which implies that $\phi_{x}=\phi_{y}$, or $x=y$.

$\rho$ is biholomorphic on $R^{1}-\rho^{-1}(0)$. Let $\pi^{0} x \neq 0$; then for $U_{x}$ a small enough neighborhood of $x,\left(U_{x}, \pi^{0}\right)$ is a local parameter at $x$. Similarly, since $\pi x$ is in $V_{\text {reg, }}\left(U_{\rho x}, \pi^{1}\right)$ is a local parameter at $x$ for $U_{\rho x}$ small enough. But since $\pi^{0}=\pi^{1} \circ \rho, \rho$ also is biholomorphic in a sufficiently small neighborhood of $x$.

Easily $\rho$ is holomorphic. For if $f$ is holomorphic on $V, f \circ \rho$ is holomorphic on $R-\rho^{-1}(0)$, but also continuous. Thus since $R^{1}$ is a complex manifold, $f \circ \rho$ is holomorphic on $R^{1}$.

Now, we can transfer the structure of $R^{1}$ to $R$, the normalization of $V$ by $\pi^{-1} \circ \rho$, making of $R$ a complex manifold. This structure coincides with the given one on $R-\pi^{-1}(0)$, and with it $\pi$ is still holomorphic. Thus the two structures do coincide and $R$ is a complex manifold. 
If $x$ in $S$ is a singular point, $\pi^{-1}(x)=\left\{r_{1}, \cdots, r_{n}\right\}$ and for $U$ a neighborhood of $x$, there are neighborhoods $U_{i}$ of $r_{i}$ such that $\pi: U U_{i}$ onto $U$ and $\pi: \cup U_{i}-\left\{r_{1}, \cdots, r_{n}\right\} \rightarrow U-\{x\}$ one-one and onto. We call the $\pi\left(U_{i}\right)$ the sheets of $S$ at $x$. Note that $\pi\left(U_{i}\right)$ is an analytic space and $U_{i}$ is its normal model. If $x$ is in $\pi\left(U_{i}\right)_{\mathrm{reg}}$, then $\pi: U_{i} \leftrightarrow \pi\left(U_{i}\right)$ is a biholomorphic map. If $x$ is in $\pi\left(U_{i}\right)_{\text {sing }}$, then $\pi$ is just holomorphic. This type of point we call a branch point of $\pi\left(U_{i}\right)$ or a branch point of $S$ if $\pi^{-1}(x)$ is just one point ( $x$ is an irreducible point). If $\pi^{-1}(x)$ is more than one point we call $x$ an identification point.

Let $x$ be a branch point of $S$; then $\pi: R \rightarrow S$ is one-one holomorphic from a neighborhood of $\pi^{-1}(x)=r$ to a neighborhood of $x$ in $S$. For the moment we replace these neighborhoods by $R$ and $S$ respectively and we assume $S$ is a variety $V$ at 0 in $C^{n}$. Then $\hat{\pi}\left(\Theta_{x}^{V}\right)$ (the transfer by $\pi$ of all functions holomorphic in a neighborhood of 0 ) is a subring of $\Theta_{r}^{R}$. In fact we have:

3.6. LEMMA. $\hat{\pi}\left(\Theta_{x}^{V}\right)$ is precisely the set of germs of functions which can be uniformly approximated in a neighborhood of $r$ by polynomials in the $\hat{\pi}\left(z_{1}\right), \cdots, \hat{\pi}\left(z_{n}\right)$. Further, $\hat{\pi}\left(\Theta_{x}^{V}\right)$ contains a power of the maximal ideal of $\Theta_{r}^{R}$.

Proof. Let $f$ be in $\hat{\pi}\left(\Theta_{x}^{V}\right)$; then $f=g \circ \pi, g$ in $\Theta_{x}^{V}$. Then, for a sufficiently small polycylinder $P^{n}$ about $x$, there is an $h$ in $H\left(P^{n}\right)$ such that $g=h \mid V$. But then $h$ is approximable by polynomials in compact neighborhoods of $x$, thus also is $g$; thus $g \circ \pi=f$ is approximable by polynomials in the $z_{j} \circ \pi=\hat{\pi}\left(z_{j}\right)$ in a neighborhood of $r$. Conversely, if $f$ is in $\Theta_{r}^{R}$, in a neighborhood of $r, f \circ \pi^{-1}$ is defined, since $\pi$ is one-one. If $f$ is approximable by polynomials in the $\hat{\pi}\left(z_{j}\right), f \circ \pi^{-1}$ is approximable in a neighborhood of $x$ on $V$ by polynomials in the $z_{j}$. But $\Theta_{x}^{V}$ is closed in the topology of local uniform convergence [5], so $f \circ \pi^{-1}$ is in $\Theta_{x}^{V}$, implying $f=f \circ \pi^{-1} \circ \pi$ is in $\hat{\pi}\left(\Theta_{x}^{V}\right)$.

Now, by 3.2, there is an ideal $\mathcal{C}$ of $\Theta_{x}^{V}$ such that $\mathfrak{e} \Theta_{r}^{R} \subset \Theta_{x}^{V}$. But $\mathfrak{e} \Theta_{r}^{R}$ is an ideal of $\Theta_{r}^{R}$; thus $\Theta_{x}^{V}$ contains an ideal $I$ of $\Theta_{x}^{R}$, and $I \neq 0$, since $\mathcal{C} \neq 0$. But the only ideals of $\Theta_{r}^{R}$ are the powers of the maximal ideal. Let $g$ be in $I$; suppose $g$ has a zero of order $n$ at $r, g^{\prime}$ has a zero of order $\geqq n$. Then $g^{\prime} / g$ is in $\Theta_{r}^{R}$, so $g^{\prime}=g\left(g^{\prime} / g\right)$ is also in $I$. Thus $I$ contains $M^{n}(M=$ maximal ideal $)$. Let $n^{0}$ $=\min \left\{n ; I \supset M^{n}\right\}$. Then $I=M^{n}$; for if not then there is a $g$ in $I$ with a zero of order $m<n^{0}$, but this implies, as we have seen, $I \supset M^{m}$, contradicting the definition of $n^{0}$.

3.7. CoRollary. If $x$ is a branch point of $S, \pi: R \rightarrow S, \pi(r)=x$, then there exists an $N$ such that, if $f$ is analytic in a neighborhood of $r$ and vanishes at $r$ with order $\geqq N$, then $f$ is in $\hat{\pi}\left(\Theta_{x}^{S}\right)$.

Now we look at the local behavior at an identification point; so we may assume we have maps $\pi_{i}:\{|z|<1\} \leftrightarrow V_{i} \subset P^{n}, \pi_{i}(0)=0$, and $V_{1}, \cdots, V_{k}$ are the irreducible components of a variety $V$ containing 0 . Let $T=\left\{\left(f_{1}, \cdots, f_{k}\right) ; f_{j}\right.$ in $\Theta_{0}^{1}$ such that there exists $g$ in $\left.\Theta_{0}^{n}, g \mid V_{j} \circ \pi_{j}=f_{j}\right\}$. 
3.8. Lemma. There are integers $N_{j} \geqq 0$ such that if $f_{j}$ is in $\Theta_{0}^{1}$ and vanishes at 0 with order $\geqq N_{j}$, then $\left(f_{1}, \cdots, f_{k}\right)$ is in $T$.

Proof. Write $V_{i}=V\left(p_{1}^{i}, \cdots, p_{n-1}^{i}\right)$. Since $V_{j} \cap V_{i}=\{0\}$, there is $p_{i}^{j}$ in $I\left(V_{j}\right)$ such that $\left.p_{i}^{j}\right|_{v_{i}}$ has its only zero at 0 . Let $n_{i j}$ be the order of vanishing of $\left.p_{i}^{j}\right|_{v_{i}} \circ \pi_{i}$ at 0 . According to 3.7 there is an integer $n_{i i}$ such that if $f$ is in $\Theta_{0}^{1}$ and vanishes at 0 with order $\geqq n_{i i}$, then $f$ is in $\hat{\pi}_{i}\left(\Theta_{0}^{n}\right)$. Let $N_{i}=\sum_{j=1}^{n} n_{i j}$. These are the required integers.

Let $\left(f_{1}, \cdots, f_{k}\right)$ be as in the statement of the lemma. Then $f_{i}^{1}=\left[f_{i}\left[\left.\prod_{j \neq i} p_{i}^{j}\right|_{v_{i}} \circ \pi_{i}\right]^{-1}\right]$ is in $\Theta_{0}^{j}$ and vanishes with order $\geqq n_{i i}$, so there is a $g_{i}$ in $\Theta_{0}^{n}$ such that $\left.g_{i}\right|_{v_{i}}=f_{i}^{1}$, or, what is the same, $f_{i}=\left(\left.g_{i} \prod_{j \neq 1} p_{i}^{j}\right|_{v_{i}}\right) \circ \pi_{i}$. But now, for $m \neq i,\left.g_{m} \prod_{j \neq m} p_{m}^{j}\right|_{v_{i}}$ vanishes on $V_{i}$, so we have

$$
f_{i}=\left(\sum_{m=1}^{k} g_{m} \prod_{j \neq m} p_{m}^{j}\right) \circ \pi_{i} .
$$

Then $g=\sum_{m=1}^{k} g_{m} I I_{j \neq m} p_{m}^{j}$ is in $\Theta_{0}^{n}, g \circ \pi_{i}=f_{i}$, proving $\left(f_{1}, \cdots, f_{n}\right)$ is in $T$.

Now consider $K$ a proper compact set on a connected one-dimensional analytic space $S$. Since $S_{\text {sing }}$ is discrete $K \cap S_{\text {sing }}$ is finite. Furthermore we can find a neighborhood $U$ of $K$ such that $\bar{U}$ is compact and $U \cap S_{\text {sing }}$ $=K \cap S_{\text {sing. }}$.

Let $(R, \pi)$ be the normalization of $S$. Then $\pi^{-1}(U)$ has finitely many connected components. For any regular point on $S$ can be connected to a singular point by an arc contained in $S_{\text {reg, }}$ the set of regular points. Since $\pi^{-1}$ is homeomorphic on $S_{\text {reg, }}$, any $r \in R-\pi^{-1}\left(S_{\text {sing }}\right)$ can be connected by an arc in $\pi^{-1}(U)$ to a point in $\pi^{-1}\left(S_{\text {sing }}\right)$, but $\pi^{-1}\left(S_{\text {sing }} \cap U\right)$ is finite.

Thus we can write $\pi^{-1}(U)=U U_{i}$, where $U_{i}$ is an open connected set on a Riemann surface $R_{i}$ (a component of $R$ ). Since $\pi$ is proper and $\bar{U}$ is compact, each $\bar{U}_{i}$ is compact, so we may consider $U_{i}$ to be an open subset of a compact Riemann surface $R_{i}^{\prime}$.

Now if $x \in K \cap S_{\text {sing }}, \pi^{-1}(x)=\left\{r_{1}, \cdots, r_{t}\right\}$. Now any $r_{i}$ may be the only point of $\pi^{-1}(K)$ on that component of $R$, for the branch corresponding to $r_{i}$ may intersect $K$ only at $x$. We want to exclude such points from $\pi^{-1}(K)$, so instead we take $\tilde{K}$, the closure of $\pi^{-1}\left(K \cap S_{\mathrm{reg}}\right)$, and thus if $K$ has no isolated points (which case we exclude) $\pi$ maps $\widetilde{K}$ onto $K$. Since $\widetilde{K}$ differs from $\pi^{-1}\left(K \cap S_{\text {reg }}\right)$ by a finite point set, we have the following: (a) if $K$ is the closure of its interior, so also is $\tilde{K}$ the closure of its interior; (b) if int $K$ is connected, int $\widetilde{K}$ is connected; (c) if $K$ is bounded by finitely many piecewise analytic curves, so is $\tilde{K}$.

Now $\tilde{K}$ is a disjoint union of finitely many proper compact sets $\tilde{K}_{i}$ on compact Riemann surfaces. Thus $A(\widetilde{K})$ obviously is the direct sum of the $A\left(\tilde{K}_{i}\right)$, and so by $2.2, S(A(\tilde{K}))=S\left(A\left(\tilde{K}_{i}\right)\right)=\bigcup \tilde{K}_{i}=\tilde{K}$.

3.9. THEOREM. Let $K$ be a compact subset of a connected one-dimensional analytic space $S$. Then there are finitely many compact Riemann surfaces 
$R_{1}, \cdots, R_{t}$ and $\tilde{K}_{i} \subset U_{i} \subset R_{i}, \tilde{K}_{i}$ compact, $U_{i}$ open, and a map $\pi: \cup U_{i}$ onto a neighborhood $U$ of $K$ such that (i) for $R=U U_{i},(R, \pi)$ is the normalization of $U$, (ii) $\tilde{K}=U \tilde{K}_{i}$ is the closure of $\pi^{-1}\left(K \cap S_{\mathrm{reg}}\right)$. (iii) Let $K \cap S_{\mathrm{sing}}=U \cap S_{\mathrm{sing}}$ $=\left\{s_{1}, \cdots, s_{n}\right\}$. Let $\pi^{-1}\left(s_{i}\right) \cap \tilde{K}=\left\{x_{i 1}, \cdots, x_{i t_{i}}\right\}$. There exist integers $n_{i j}$ such that $A(K) \supset\left\{f \circ \pi^{-1} ; f\right.$ holomorphic in a neighborhood of $\tilde{K}, f\left(x_{i p}\right)=f\left(x_{i q}\right)$ for all $p, q, 1 \leqq i \leqq n, f(x)-f\left(x_{i j}\right)$ has a zero of at least order $n_{i j}$ at $\left.x_{i j}\right\}$. (iv) In particular, $A(K) \supset\left\{f \circ \pi^{-1} ; f\right.$ in $\left.I\right\}$ where $I$ is an ideal of $A(K)$ with finite hull; in fact hull $I=\pi^{-1}\left(K \cap S_{\text {sing }}\right)$.

Proof. If in the preceding discussion we take $\tilde{K}_{i}=\tilde{K} \cap U_{i}$, then (i), (ii) are verified. As for (iii), the integers $n_{i j}$ are just those given by Lemma 3.8 applied at the point $s_{i}$. That is, if $\pi^{-1}\left(s_{i}\right)=\left\{x_{i 1}, \cdots, x_{i t_{i}}\right\}$, then for $S_{j}$ a branch of $S$ at $s_{i}$, let $n_{i j}$ be the integer given by Lemma 3.8, so that if $f_{j}$ is in $\Theta_{x_{i j}}^{R}$ and vanishes at $x_{i j}$ with order $\geqq n_{i j}$, then there is a $g \in \Theta_{s_{i}}^{n}$ (where we now consider a neighborhood of $s_{i}$ as embedded in $\left.\mathfrak{C}^{n}\right)$ such that $\left(g \mid S_{j}\right) \circ \pi=f_{j}$. Now let $f$ be in $H\left(V, \Theta^{R}\right), V$ a neighborhood of $\tilde{K}$; suppose $f$ is as described in (iii). Since $f$ identifies the points which $\pi$ identifies, $f \circ \pi^{-1}$ is a continuous function on $K$. Since $S_{\text {sing }} \cap K=S_{\text {sing }} \cap U, g=f \circ \pi^{-1}$ is a continuous function on $\pi(V)$ and since $\pi \mid R-\pi^{-1}\left(S_{\text {sing }}\right)$ is biholomorphic, for all regular points $s$ we have $g_{s} \in \Theta_{s}^{S}$. If $s=s_{i}$, then by Lemma 3.8, since $f$ is as described in (iii), there is a $G \in \Theta_{s_{i}}^{n}$ such that $G\left|S_{j}=g-g\left(s_{i}\right)\right| S_{j}$; so $g=G+g\left(s_{i}\right)$. But $G+g\left(s_{i}\right)$ is in $\Theta_{s_{i}}^{n}$, so $g$ is in $\Theta_{s,}^{S}$. Thus $g$ is in $H\left(\pi(V), \Theta^{S}\right)$, so that $g\left|K=f \circ \pi^{-1}\right| K$ is in $A(K)$. Thus (iii) is proven.

Letting $H(\tilde{K})=[f$ holomorphic in a neighborhood of $\tilde{K}]$, then by (iii), $A(K) \supset\left[f \circ \pi^{-1} ; f\right.$ in $\left.I\right]$ where $I=\left[f\right.$ in $H(\tilde{K}) ; f\left(x_{i j}\right)=0, f$ has a zero of order $\geqq n_{i j}$ at $\left.x_{i j}\right]$ is an ideal of $H(\tilde{K})$. Now $H(\widetilde{K})$ is dense in $A(\tilde{K})$. Thus $\bar{I}$ (closure of $I$ in $A(\tilde{K}))$ is also an ideal of $A(\tilde{K})$, and since $A(K)$ is closed, $A(K)$ $\supset\left\{f \circ \pi^{-1} ; f\right.$ in $\left.\bar{I}\right\}$. Now hull $\bar{I}=\left\{x_{i j}\right\}$. Surely each $x_{i j}$ is in hull $\bar{I}$. On the other hand, if $x$ is in $\tilde{K}, x \neq x_{i j}$, by 2.3 , there is an $f_{i j}$ in $H(\tilde{K})$ such that $f_{i j}(x) \neq 0, f_{i j}\left(x_{i j}\right)=0$. Then $f=\left[\prod f_{i j}\right]^{\text {sup } n_{i i}}$ is in $I$ and $f(x)=0$, so $x$ is not in hull $\bar{I}$.

\subsection{ThEOREM. $S(A(K))=K$.}

Proof. Since $A(K)$ is an algebra of continuous functions on $K$, evaluation at any point of $K$ is a continuous homomorphism of $A(K)$. We prove $A(K)$ separates point of $K$. Let $s, t$ be in $K, s$ in $S_{\text {reg. }}$. Let $x=\pi^{-1}(s)$. There is a function $f_{t k}$ in $H(\tilde{K})$ such that $f_{t k}(x) \neq 0, f_{t k}\left(y_{k}\right)=0$, for all $y_{k}$ in $\pi^{-1}(t)$. There is an $f_{i j}$ in $H(\tilde{K})$ such that $f_{i j}(x) \neq 0, f_{i j}\left(x_{i j}\right)=0$. Then $f=\left[\prod f_{t k} f_{i j}\right]^{\text {sup } n_{i i}}$ is in $I$ and $f(x) \neq 0, f\left(\pi^{-1} t\right)=0$. Thus $g=f \circ \pi^{-1}$ is in $A(K)$ and $g(s) \neq g(t)$.

Suppose now $s=s_{1}, t=s_{2}$. By 2.3 there is an $f$ in $M(\tilde{K}), f\left(x_{1 j}\right)=1, f\left(x_{i j}\right)=0$ if $i \neq 1$, and $f(x)-f\left(x_{i j}\right)$ has a zero of order at least $n_{i j}$ at $x_{i j}$. Then $f$ is in $H(K)$ and by (iii) of 3.9, $g=f \circ \pi^{-1}$ is in $A(K)$. But $g(s)=1, g(t)=0$. Thus $A(K)$ separates points. 
Let $h$ be in $S(A(K))$, suppose $h$ is not evaluation at any $s_{1}, \cdots, s_{n}$. Then there is an $f_{1}$ in $A(K)$ such that $f_{1}\left(s_{j}\right)=0,1 \leqq j \leqq n$, and $h\left(f_{1}\right)=1$. For $f$ in $\vec{I}$, let $\tilde{h}(f)=h\left(f \circ \pi^{-1}\right)$. $\tilde{h}$ is a nonzero continuous homomorphism on $\bar{I}$, since $\left[f_{1} \circ \pi\right]^{N}$, where $N=\sup \left\{n_{i j}\right\}$ is in $\bar{I}$, and $\tilde{h}\left(\left(f_{1} \circ \pi\right)^{N}\right)=h\left(\left(f_{1}\right)^{N}\right)=1$. But now, by $2.2 S(A(\tilde{K}))=\tilde{K}$, and hull $\bar{I}=\left\{x_{i j}\right\}$, so $S(\bar{I}) \equiv K-\left\{x_{i j}\right\}$. Then $\tilde{h}(f)=f(x)$ for some $x$ in $K, x \neq x_{i j}$. Thus $\pi x$ is a regular point of $S$. Let $g$ be in $A(K)$; we prove $h(g)=g(\pi x)$. Then, since $\left(f_{1}\right)^{N} \circ \pi$ is in $I, g\left(f_{1}\right)^{N} \circ \pi=(g \circ \pi)\left(\left(f_{1}\right)^{N} \circ \pi\right)$ is in $I$, so that

$$
\begin{aligned}
h(g) & =h(g) h\left(\left(f_{1}\right)^{N}\right)=h\left(g\left(f_{1}\right)^{N}\right)=h\left(g\left(f_{1}\right)^{N} \circ \pi\right) \\
& =g(\pi x)\left(\left(f_{1}\right)^{N}(\pi x)\right)=g(\pi x) h\left(\left(f_{1}\right)^{N} \circ \pi\right)=g(\pi x) .
\end{aligned}
$$

Then $S(A(K))=K$.

4. Runge sets. If $K$ is a compact set on an analytic space $S$, it is not necessarily true that $A(K)=C(K) \cap H$ (int $K$ ), i.e., it is not always true that every function continuous on $K$ and analytic in the interior of $K$ is approximable by functions holomorphic in a neighborhood of $K$. If $S$ is a complex manifold, it is evident that $A(K) \subset C(K) \cap H$ (int $K$ ) since $H$ (int $K$ ) is closed in the topology of uniform convergence. But for $S$ a general analytic space this has only recently been proven by Remmert and Grauert [5]. We have already used this result in 3.6. The possibility of $A(K)=C(K) \cap H$ (int $K$ ) depends greatly on the geometry of $K$.

Definition. If $K \subset S$ is a proper compact subset of $S$ and there is a domain $U \supset K$ such that $K$ is $H(U)$-convex, we call $K$ a $R$ unge set.

4.1. Theorem (Runge's Theorem) (Behnke). Let $R$ be a Riemann surface, $U \subset U^{\prime}$ domains on $R$. Suppose $U$ is simply connected with respect to $U^{\prime}$, i.e., if $\gamma \subset U$ bounds a domain in $U^{\prime}$ it bounds a domain in $U$. Then every function holomorphic on $U$ can be approximated uniformly on compact sets by functions holomorphic on $U^{\prime}[3]$.

4.2. CoROLlaRY. Let $K$ be a compact, proper subset of $R$. If there is a family of domains $U(n) \subset U, n$ an integer, satisfying (i) $U(n)$ is simply connected with respect to $U(1)$, (ii) $U(n) \supset U(n+1)$, (iii) $\bigcap_{n} U(n)=K$, then $H(U(1))$ is dense in $A(K)$.

Proof. Let $f$ be in $A(K) ; f$ can be uniformly approximated by functions holomorphic in a neighborhood of $K$. If $U$ is a neighborhood of $K$ there is an $n$ such that $U(n) \subset U$. If $f$ is in $H^{0}\left(U, \Theta^{R}\right)$, then $\left.f\right|_{U(n)}$ is uniformly approximable on $K$ by functions in $H(U(1))$. Thus the corollary is proven.

If $K$ has a neighborhood $U$ such that $H(U)$ is dense in $A(K)$, then $K$ is $H(U)$-convex. For we know that $S(A(K))=K$, so it follows that $S(H(U))$ $=K$, with the sup norm on $K$ on $H(U)$. Thus if $x \in U-K$, the map $f \rightarrow f(x)$ is not a continuous multiplicative functional on $H(U)$ in this norm, so there is an $f \in H(U)$ such that $|f(x)|>\|f\|_{k}$. But this is the condition of $H(U)$ convexity. 
It is easily seen that if $K=\Omega \cup \gamma_{1} \cup \ldots \cup \gamma_{n}, \Omega$ a domain, $\gamma_{1}, \cdots, \gamma_{n}$ piecewise analytic curves, then the hypotheses of 4.2 are satisfied with $U(1)$ a sufficiently small neighborhood of $K$, so $K$ is a Runge set.

4.3. Corollary. Let $K$ be a Runge set in a compact Riemann surface $R$. There is a neighborhood $U$ of $K$ such that $H(U)$ is dense in $C(K) \cap H$ (int $K$ ), and in particular $A(K)=C(K) \cap H($ int $K)$.

This follows from an immediate application of Bishop's theorem, Theorem 1.2. We now obtain the same result for Runge sets on one-dimensional analytic spaces.

4.4. Theorem. Let $K$ be a compact subset of $S$, and $\tilde{K}$ as defined in $\$ 3 . K$ is a Runge set if and only if $\tilde{K}$ is a Runge set. If $K$ is a Runge set, $A(K)=C(K)$ $\cap H($ int $K)$.

Proof. If $K$ is a Runge set, there is a $U \subset S$ such that $U \supset K$ and $K$ is $H(U)$-convex. If $K \subset V \subset U$, then obviously $K$ is also $H(V)$-convex, so we may assume $U$ chosen so small that $\pi^{-1}(U) \cap \pi^{-1}\left(S_{\text {sing }}\right)=\tilde{K} \cap \pi^{-1}\left(S_{\text {sing }}\right)$. Then if $x$ is in $\pi^{-1}(U)$, but not in $\tilde{K}$, then $\pi x$ is not in $K$, so there is an $f \in H(U)$ such that $|f(\pi x)|>\|f\|_{K}$. Then $f \circ \pi$ is holomorphic on $\pi(U)$ and $|f \circ \pi(x)|$ $>\|f \circ \pi\|_{\tilde{K}}$. Thus $\tilde{K}$ is $H\left(\pi^{-1}(U)\right)$-convex. Now we can write $\tilde{K}=U \tilde{K}_{i}$, a disjoint union and $\pi^{-1}(U)=\bigcup \tilde{U}_{i}$ where $\tilde{U}_{i} \supset \tilde{K}_{i}$ are both subsets of a compact Riemann surface. Obviously $\tilde{K}_{i}$ is $H\left(\tilde{U}_{i}\right)$-convex, so it follows from 4.3 that $A\left(\tilde{K}_{i}\right)=C\left(\tilde{K}_{i}\right) \cap H\left(\right.$ int $\left.\tilde{K}_{i}\right)$, and then $A(\tilde{K})=C(\tilde{K}) \cap H($ int $\tilde{K})$.

Now we assume only $\tilde{K}$ is a Runge-set, and is $H(\tilde{U})$-convex.

Let $K \cap S_{\text {sing }}=\left\{s_{1}, \cdots, s_{n}\right\}$, let $s_{1}, \cdots, s_{e}$ be in $\partial K, s_{e+1}, \cdots, s_{n}$ in int $K$. Let $\pi^{-1}\left(s_{i}\right)=\left\{x_{i j}, 1 \leqq j \leqq k_{i}\right\}$, and $n_{i j}$ the integer corresponding to $s_{i}$ as 3.9. Let $I=\left\{f\right.$ in $H(\tilde{U}) ; f$ has a zero of at least order $n_{i j}$ at $\left.x_{i j}\right\}$. Now $\tilde{K}$ is $I$-convex. For $I$ is an ideal in $H(\tilde{U})$ and since $\tilde{K}$ is $H(\tilde{U})$-convex, the closure of $H(\tilde{U})$ on $\tilde{K}$ is $A(\tilde{K})$, and $S(A(\tilde{K}))=\tilde{K}$. Thus the closure $\bar{I}$ of $I$ is an ideal of $A(\tilde{K})$, so $S(\bar{I})$ is a subspace of $\tilde{K}$. But if $\tilde{K}$ is not $I$-convex, there is an $x$ in $\tilde{U}-\tilde{K}$ such that $|f(x)| \leqq\|f\|_{K}$ for all $f$ in $I$, so evaluation at $x$ extends to a homomorphism of $\bar{I}$, not evaluation at any point of $\tilde{K}$; since $I$ separates points of $\tilde{U}$ (but for hull $I \subset \tilde{K}$ ), contradicting $S(\bar{I}) \subset \tilde{K}$. Since $I \subset[f \circ \pi$; in $H(\pi(\tilde{U}))]$, then $K$ is $H(\pi(\tilde{U}))$-convex.

Now, by Bishop's theorem, 1.2, $\bar{I}$ contains $\{f$ in $C(K) \cap H$ (int $K$ ) such that $f$ vanishes on $\pi^{-1}\left(S_{\text {sing }}\right)$ and has a zero of order $\geqq N$ at $\left.x_{i j}, i>e\right\}$, for some integer $N$. Let $g$ be the ideal of $A(\tilde{K}), \mathfrak{g}=\left\{f\right.$ in $A(\tilde{K}) ; f(x)=f\left(x_{i j}\right)$ has a zero of order $\geqq N$ for $i>\boldsymbol{e}\}$.

Now let $f$ be in $C(K) \cap H\left(\right.$ int $K$ ) such that $f$ vanishes on $S_{\text {sing }} \cap \partial K$; then $f \circ \pi$ is in $C(\tilde{K}) \cap H($ int $\tilde{K})=A(\tilde{K})$, since $\tilde{K}$ is a Runge set. By 2.4 considering only the points $x_{i j}, i>e$, we can write $f \circ \pi=g+h, g$ is meromorphic on $R$, holomorphic on $K$, and $h$ is in $\mathscr{g}$. We can arrange, in fact, that $h$ is in $\bar{I}$ by adding and subtracting a meromorphic function in $g$ which takes the values $g\left(x_{i j}\right)$ at $x_{i j}, i<e$; then $h$ vanishes on $\pi^{-1}\left(S_{\mathrm{sing}}\right)$. Since $g$ is holomorphic in a 
neighborhood of $\tilde{K}$, we can write $g=g_{1}+g_{2}$, where $g_{1}, g_{2}$ are also meromorphic functions, holomorphic on $\tilde{K}$, such that $g_{1}$ vanishes with order $n_{i j}$ at $x_{i j}$, $i \leqq e$, and $g_{2}$ is in $\mathscr{g}$, i.e., let $g_{2}$ be in $\mathscr{g}$ and $g_{2}$ have the same power series up to the $n_{i j}$ term as $-g$ at $x_{i j}$ (in some local coordinate system) for all $i \leqq e$. This can be done by 2.3. Also $g_{1}, g_{2}$ vanish on $\pi^{-1}\left(S_{\text {sing }}\right)$. Thus we have $f \circ \pi=g_{1}$ $+\left(g_{2}+h\right) ; g_{1}$ vanishes of order $n_{i j}$ at $x_{i j}, i \leqq e$, and $g_{2}+h$ is in $g$ and vanishes on $\pi^{-1}\left(S_{\text {sing }}\right)$. Thus $g_{2}+h$ is in $\bar{I}$ and $\left\{F \circ \pi^{-1} ; F\right.$ in $\left.\bar{I}\right\} \subset A(K)$, so $\left(g_{2}+h\right) \circ \pi^{-1}$ is in $A(K)$, so also is in $C(K) \cap H$ (int $K)$. Thus $g_{1} \circ \pi^{-1}=f-\left(g_{2}+h\right) \circ \pi^{-1}$ is also in $C(K) \cap H$ (int $K$ ). But $g_{1}$ is meromorphic on $R$, and holomorphic on $\tilde{K}$, so is holomorphic in a neighborhood $W$ of $\tilde{K}$. Thus $g_{1} \circ \pi^{-1} \mid S_{\text {reg }}$ is in $H\left(\pi(W) \cap S_{\mathrm{reg}}\right)$. But for $x_{i j}, i \leqq e, g_{1}$ vanishes of order $n_{i j}$, so by $3.8\left[g_{1} \circ \pi^{-1}\right]_{s_{i}}$ is in $\Theta_{s_{i}}^{S}$. This, for all $i \leqq e$, but for $i>e, g_{1} \circ \pi^{-1}$ is in $\Theta_{s_{i}}^{S}$ since $s_{i}$ is in int $K$. Thus $g_{1} \circ \pi^{-1}$ is in $H\left(\pi(W), \Theta^{S}\right), \pi(W)$ is a neighborhood of $K$, so $g_{1} \circ \pi^{-1}$ is in $A(K) .\left(g_{2}+h\right) \circ \pi^{-1}$ is in $A(K)$ also, thus $f=g_{1} \circ \pi^{-1}+\left(g_{2}+h\right) \circ \pi^{-1}$ is in $A(K)$.

Since $\partial K \cap S_{\text {sing }}=\left\{s_{1}, \cdots, s_{e}\right\}$ is finite, given any complex numbers $t_{1}, \cdots, t_{e}$, there is a $g$ in $A(K)$ such that $g\left(s_{i}\right)=t_{i}$. Thus if $f$ is in $C(K)$ $\cap H$ (int $K)$, there is a $g$ in $A(K)$ such that $g\left(s_{i}\right)=f\left(s_{i}\right)$. Thus $f-g$ is in $C(K) \cap H$ (int $K$ ) and vanishes on $\partial K \cap S_{\text {sing }}$, so by the above is in $A(K)$. Thus $f=(f-g)+g$ is also in $A(K)$. Theorem 4.4 is proven.

We obtain as a corollary of the above (more explicitly, corollaries of Bishop's Theorem (1.2)) certain approximation theorems; more general results are found in [13].

4.5. TheOREM. Let $\gamma$ be a piecewise analytic arc on a one-dimensional analytic space $S$. Then $A(\gamma)=C(\gamma)$, i.e., the algebra of functions holomorphic in a neighborhood of $\gamma$ is dense in $C(\gamma)$.

Proof. Let $R$ be the normalization of $S, \pi: R \rightarrow S$, and let $\tilde{\gamma}=\pi^{-1}\left(\gamma \cap S_{\mathrm{reg}}\right)$. Then $\tilde{\gamma}$ is a piecewise analytic arc on $R$, and is obviously a Runge set. Then $\gamma$ is a Runge set and 4.4 applies, so $A(\gamma)=C(\gamma)$, since int $\gamma$ is empty.

Now since the image under a holomorphic map of a domain in $C^{1}$ into $C^{n}$ is always an analytic space [9], we have

4.6. Theorem. Let $\gamma$ be any analytic arc in $C^{n}$ (i.e., the image of $[0,1]$ under a holomorphic homeomorphism). Then $A(\gamma)=C(\gamma)$.

4.7. TheOREm. Let $\gamma$ be a piecewise analytic arc on a variety $V$ in a polynomial convex subset $U$ of $C^{n}$. Then the polynomials are dense in $C(\gamma)$.

Proof. Let $R$ be the normalization of $V, \pi: R \rightarrow S$, and let $\tilde{\gamma}=\pi^{-1}\left(\gamma \cap S_{\text {reg }}\right)$. We can easily write $\tilde{\gamma}$ as the decreasing intersection of domains which are simply connected in $R$. Thus, as in 4.2, $\tilde{\gamma}$ is $H(R)$-convex, and similarly, as in 4.4, this implies $\gamma$ is $H(V)$-convex. But then $\gamma$ as a subset of $U$ is $H(U)$ convex. For if $x \in U-\gamma$ and $x \in V$, there is an $f \in H(V)$ such that $|f(x)|>\|f\|_{\gamma}$. But $f$ is just the restriction to $V$ of a function $g \in H(U)$, so $|g(x)|>\|g\|_{\gamma}$. If 
$x \notin V$, then there is an $f \in H(U)$ such that $f \mid V=0$, and $f(x) \neq 0$. Then $|f(x)|$ $>\|f\|_{\gamma}$. Then, since $\gamma$ is $H(U)$-convex, $H(U)$ is dense in $A(\gamma)$. But $U$ is polynomial convex, implying that the polynomials are dense in $H(U)$ in the topology of uniform convergence on compact subsets of $U$, so the polynomials are dense in $A(\gamma)$. But by $4.5, A(\gamma)=C(\gamma)$.

5. The maximality theorem. Before proceeding to the extension of Wermer's maximality theorem we prove a general Banach algebra result which appears in another form in [7].

Definition. Let $A$ be a function algebra on $X$. Let $Y \subset S(A) . A$ is $(Y, X)$ maximal if and only if, for any algebra $B, A \subset B \subset C(X), S(B) \supset Y$ implies $B=A$. We say $A$ is a maximal subalgebra of $C(X)$ if $A$ is $(X, X)$-maximal. This is the same as the definition which appears in [11].

5.1. Theorem. Let $M$ be a maximal subalgebra of $C(X)$. Let $A \subset M$ be a closed subalgebra of $M$ such that $A \supset I, I$ an ideal of $M$. Let $H$ be the hull of $I$ in $S(M), g=\{f$ in $C(X)$ vanishing on $H \cap X\}$. Then if $B$ is a closed subalgebra of $C(X)$, and $B \supset A$, then either $B \subset M$ or $B \supset$ g.

Proof. Suppose there exists $g$ in $B-M$. Let $P=\{$ polynomials in $g$, coefficients in $M\}$; then $\mathcal{P}$ is dense in $C(X)$. Let $h$ be in $C(X), p_{n}$ in $\mathcal{P}$, such that $p_{n}$ converges uniformly to $h$. Then for $f$ in $I, f p_{n}$ converges to $f h$. Since $f p_{n}$ is in $B, f h$ is in $B$. Thus $B$ contains $\{f h ; f$ in $I, h$ in $C(X)\}$. But then $B$ contains the closed algebra generated by this set, which is a closed ideal of $C(X)$, obviously, $\mathfrak{g}$, since it has the same hull as $g$.

5.2. Corollary. Let $M$ be a maximal subalgebra of $C(X)$. Let $H_{1}, \cdots, H_{n}$ be pairwise disjoint hulls in $S(M)$. Let $A=\left\{f \in M ; f \mid H_{j}\right.$ is constant, $j=1, \cdots, n\}$. Then $A$ is a maximal subalgebra of $C(\Gamma(A))(\Gamma(A)$ is the Šilov boundary of $A)$, among all subalgebras having $S(A)$ as space of maximal ideals, i.e., $A$ is $(S(A), \Gamma(A))$-maximal.

Proof. $A$ is a subalgebra of $M$, thus there exists a map $\pi: S(M) \rightarrow S(A)$. Obviously $\pi$ is a homeomorphism on $S(M)-\left(\bigcup_{i=1}^{n} H_{i}\right)$ and $\pi\left(H_{i}\right)=y_{i}$, a single point, and $\pi(x) \supset \Gamma(A)$. Let $B \subset C(\Gamma(A))$, and $S(B)=S(A)$. $B$ is a closed subalgebra of $C(X)$; thus either $B C M$, or $B \supset\left\{f\right.$ in $\left.C(X) ; f \mid\left(U H_{i}\right) \cap X=0\right\}$, since $\cup H_{i}$ is a hull, and we can apply the theorem. If $B \subset M$, since $S(B)=S(A)$, any $f$ in $B$ is such that $f \mid H_{j}=f\left(y_{j}\right)=$ constant. Thus $B C A$, so $B=A$. If not, then $B$, as a subalgebra of $C(\Gamma(A))$, contains $\left\{f\right.$ in $C(\Gamma(A)) ; f\left(y_{i}\right)=0$ for $y_{i}$ in $\Gamma(A)\}$. But then, since $B$ separates $\Gamma(A)$, obviously $B=C(\Gamma(A))$.

5.3. Corollary. Let $M$ be a maximal subalgebra of $C(X)$. Let $H_{1}, \cdots, H_{n}$ be disjoint hulls in $S(M)$, with $H_{j} \cap X \neq \varnothing ; j=1, \cdots, n$. Then $A=\{f$ in $M$; $\left.f\right|_{H_{j} \cap X}$ is constant, $\left.j=1, \cdots, n\right\}$ is a maximal subalgebra of $C(\Gamma(A))$.

Proof. We calculate the space of maximal ideals as above. Letting $H_{j}^{\prime}=$ hull (kernel $\left(H_{j} \cap X\right)$ ) we have $\pi: S(M) \rightarrow S(A)$ onto, $\pi: S(M)-\bigcup_{t-1}^{n} H_{j}^{\prime}$ 
is a homeomorphism, $\pi\left(H_{i}\right)=y_{i}, \pi(X)=\Gamma(A)$. Let $B$ be a subalgebra of $C(\Gamma(A)), B \supset A$. If $B \subset M$, since also $B \subset C(\Gamma(A))$, we must have for all $f$ in $B,\left.f\right|_{H_{j^{\prime}}}=$ constant. Then, by definition of $A, B \subset A$, so $B=A$. If $B \nsubseteq M$, then as in $5.2, B=C(\Gamma(A))$.

5.4. Corollary. Let $M$ be a maximal subalgebra of $C(X), A$ a subalgebra of $M$. Let $B \supset A$ be a subalgebra of $C(X)$. If $A$ contains an ideal $I$ of $M$ such that (hull $I) \cap X$ is a finite point set, then $B \subset M$ or $B=C(S(B))$.

Proof. Let (hull $I) \cap X=\left\{x_{1}, \cdots, x_{n}\right\}$. Then if $B$ is not contained in $M$, by the theorem, $B \supset\left\{f \in C(X) ; f\left(x_{i}\right)=0, i=1, \cdots, n\right\}$. Since the Silov boundary of $B$ is an identification space of $X$, we can say that for points $a_{1}, \cdots, a_{k}$ in $S(B), B \supset\left\{f \in C(S(B)) ; f\left(a_{i}\right)=0\right\}$. Since $B \mid\left\{a_{i}\right\}$ is separating, it is $C\left(\left\{a_{i}\right\}\right)$. Then if $f$ is in $B$, there exists a $g$ in $B$ such that $g\left(a_{i}\right)=\left(f\left(a_{i}\right)\right)^{-}$. Then $\bar{f}-g$ is in $C(S(B))$ and is 0 on $\left\{a_{i}\right\}$, so is in $B$. Then $\bar{f}=\bar{f}-g+g$ is in $B$. Thus with every $f$ in $B$, its conjugate $\bar{f}$ also is in $B$; thus by the StoneWeierstrass theorem, $B=C(X)$.

5.5. TheOREM. Let $S$ be a connected one-dimensional analytic space, let $K$ be compact, $K=\Omega \cup \gamma_{1} \cup \cdots \cup \gamma_{n}, \Omega$ a domain, $\gamma_{1}, \cdots, \gamma_{n}$ piecewise analytic curves.

(A) $A(K)$ is a maximal algebra in $C(\partial K)$ if and only if int $K=\Omega$ is a complex manifold.

(B) In any case, $A(K)$ is contained in precisely one maximal algebra $M$, and if $B$ is a subalgebra of $C(\partial K), B \supset A(K)$, then $B$ is dense in $C(\partial K)$ or $B \subset M$.

Proof. Let $R$ be the normal model for a neighborhood $U$ of $K, \pi: R \rightarrow U$ the projection, so that $R=\bigcup_{i=1}^{k} U_{i}$, where $U_{i}$ is a domain on a compact Riemann surface $R_{i}$. Let $\tilde{K}$ be as previously defined. Then $\tilde{K}$ is compact, and $\tilde{K}=\tilde{\Omega} \cup \tilde{\gamma}_{1} \cup \cdots \cup \tilde{\gamma}_{n}$, where $\tilde{\Omega}$ is open and $\tilde{\gamma}_{i}$ are piecewise analytic curves, $\partial \tilde{K}=\tilde{\gamma}_{1} \cup \ldots \cup \tilde{\gamma}_{n}$.

(1) We first prove the necessity of (A). Suppose $A(K)$ is a maximal subalgebra of $C(\partial K)$. Let $\left\{s_{1}, \cdots, s_{n}\right\}=K \cap S_{\text {sing }}$, and $\pi^{-1}\left(s_{i}\right)=\left\{x_{i j} ; 1 \leqq j \leqq t_{i}\right\}$. Let $B=\left\{(f \mid \partial K) \circ \pi^{-1} ; f\right.$ in $A(\tilde{K}), f\left(x_{i j}\right)=f\left(x_{i k}\right)$ for $s_{i}$ in $\left.\partial K\right\}$. Then $B$ is a closed subalgebra of $C(\partial K)$, and since $\pi$ is holomorphic, $B \supset A(K)$. Further, since every $f$ in $B$ has a holomorphic extension into $\tilde{\Omega}, B \neq C(\partial K)$. Thus, since by assumption $A(K)$ is maximal, $B=A(K)$. Now, for $x \neq y \in$ int $\tilde{K}$, there is a meromorphic function $f$ on $R$, holomorphic on $\tilde{K}$, such that $f\left(x_{i j}\right)=0$ for $s_{i}$ in $\partial K$ and $f(x) \neq f(y)$ (by 2.3). Thus, since $f \circ \pi^{-1}$ is in $B=A(K)$, there is a $g$ in $A(K)$ such that $g \circ \pi=f$. Thus $g(\pi x) \neq g(\pi y)$, so $\pi x \neq \pi y$. Thus $\pi: \tilde{\Omega} \rightarrow \Omega$ is one-one, thus is a homeomorphism, so $\Omega$ has no identification points. Now let $s$ be in int $K, \pi^{-1}(s)=x$ and $g$ in $A(\tilde{K})$ such that $g\left(x_{i j}\right)=0$ for $s_{i}$ in $\partial K, g(x)=0$ and $d g(x) \neq 0$. Then $g \circ \pi^{-1}$ is in $B=A(K)$; thus there is an $f$ in $A(K)$ such that $f \circ \pi=g$. Now for some neighborhood $\tilde{U}$ of $x, g: \tilde{U} \leftrightarrow D$, a disc with center 
the origin, so $f: \pi(\widetilde{U})=U \leftrightarrow D$ is a holomorphic homeomorphism. But $f$ is biholomorphic in $U$. For let $u$ be in $\Theta_{t}^{S}, t$ in $U$; then $\hat{\pi}(u)$ is in $\Theta_{\pi^{-1}(t)}^{R}$, and thus since $g$ is biholomorphic, $\hat{g}^{-1}(\hat{\pi}(u))$ is in $\Theta_{\ell\left(\pi^{-1}(t)\right)}^{1}$, i.e., $\hat{f}(u)$ is in $\Theta_{f(l)}^{1}$. Then $(U, f)$ is a local parameter at $s$ mapping $U$ onto a manifold; this for all $s$ in $\Omega$, so $\Omega$ must be a manifold.

(2) We now prove the sufficiency of (A), i.e., we assume $\Omega$ is a manifold. Then $\Omega \cap S_{\text {sing }}=\varnothing$. Let

$$
\left\{s_{1}, \cdots, s_{n}\right\}=K \cap S_{\mathrm{sing}}=\partial K \cap S_{\mathrm{sing}} \text {, and } \pi^{-1}\left(s_{i}\right)=\left\{x_{i j}\right\} .
$$

Since $K$ is a Runge set, by $4.4, A(K)=C(K) \cap H$ (int $K$ ), and also $\tilde{K}$ is a Runge set, so $A(\tilde{K})=C(\tilde{K}) \cap H$ (int $\tilde{K}$ ), which is (by Wermer's maximality theorem) a maximal subalgebra of $C(\partial \tilde{K})$. Then by $5.3, M=\{f$ in $A(K)$; $\left.f\left(x_{i j}\right)=f\left(x_{i k}\right), 1 \leqq i \leqq n\right\}$ is a maximal subalgebra of $C(Y)$, where $Y=\partial \tilde{K}$ with these points $\left\{x_{i 1}, \cdots, x_{i n_{i}}\right\}$ identified for $1 \leqq i \leqq n$. But then $Y$ is homeomorphic to $K$ via $\pi^{-1}: Y \leftrightarrow \partial K$, so $\left\{f \circ \pi^{-1} ; f\right.$ in $\left.A(\tilde{K}), f\left(x_{i j}\right)=f\left(x_{i k}\right), 1 \leqq i \leqq n\right\}$ is a maximal subalgebra of $C(\partial K)$. But this is just $A(K)$. For surely it contains $A(K)$. Conversely, if $f$ is in $A(\tilde{K})$, and $f\left(x_{i j}\right)=f\left(x_{i k}\right), 1 \leqq i \leqq n$, then $f \circ \pi^{-1}$ is in $C(K)$. Further, $\pi: \tilde{\Omega} \leftrightarrow \Omega$ is biholomorphic (since $\Omega \subset S_{\text {reg }}$ ), so $f \circ \pi^{-1} \mid \Omega$ is in $H(\Omega)$. But $A(K)=C(K) \cap H(\Omega)$, then $f \circ \pi^{-1}$ is in $A(K)$. Thus $A(K)$ is a maximal subalgebra of $C(\partial K)$.

(3) We now prove (B). Let $\left\{s_{1}, \cdots, s_{e}\right\} \subset \partial K,\left\{s_{e+1}, \cdots, s_{n}\right\} \subset$ int $K$. Let $A^{1}=\left\{f \in A(\tilde{K}) ; f\left(x_{i j}\right)=f\left(x_{i k}\right), 1 \leqq i \leqq e\right\}$. Then by $5.3, A^{1}$ is a maximal subalgebra of $C(Y)$ with $Y=\partial \tilde{K}$ with $\left\{x_{i 1}, \cdots, x_{i n_{i}}\right\}$ identified for $1 \leqq i \leqq e$. But then, as in the above $Y$ is $\partial K$ and $M=\left\{f \circ \pi^{-1} ; f \in A^{1}\right\}$ is a maximal subalgebra of $C(\partial K)$. As we have observed, $\{f \circ \pi ; f \in A(K)\}$ contains an ideal of $A(\tilde{K})$ whose hull is a finite point set. Thus $A(K)$ contains an ideal of $M$ whose hull is a finite point set. Then by 5.4 , if $B C C(\partial K)$ and $B \supset A(K)$, then either $B=C(S(B))$ or $B \subset M$. In the former case, since $A(K)$ separates points on $\partial K$, so does $B$, so $S(B)=\partial K$. Thus Theorem 5.5 is proven.

6. Extension of Wermer's theorem. In order to prove Theorem 6.7 it is necessary to appeal to several theorems of Oka.

6.1. Theorem (Abbildungsatz). Let $S_{1}, S_{2}$ be analytic spaces and $g$ a proper holomorphic map of $S_{1}$ into $S_{2}$. Suppose also that $g^{-1}(x)$ is finite for all $x \in X_{2}$. Then $g\left(S_{1}\right)$ is a variety in $S_{2}$ [8].

6.2. Theorem. Let $K$ be a polynomial-convex subset of $C^{n}$. Then the polynomials are dense in $A(K)[5]$.

6.3. THEOREM. Let $V$ be a variety in the $H(U)$-convex domain $U$ in $C^{n}$. Let $f \in H\left(V, \Theta^{v}\right)$. Then there is a $g \in H\left(U, \Theta^{n}\right)$ such that $g \mid V=f[5]$.

We shall also use the following lemmas concerning various types of convexities. 
6.4. Lemma. Let $V$ be a variety in a polynomial convex domain $P$ in $C^{n}$, $K \subset V$ a compact $H\left(V, \Theta^{v}\right)$-convex set. Then $K$ is polynomial convex.

Proof. Let $x \notin K$. If $x$ is not in $V$, there is an $f H\left(P, \Theta^{n}\right)$ such that $f(x)=1$, $f \mid V=0$. If $x$ is in $V$, there is an $f$ in $H\left(V, \Theta^{V}\right)$ such that $f(x)=1$ and $\|f \mid K\|<\epsilon$. But by $6.3 f=g \mid V ; g H\left(P, \Theta^{n}\right)$, so $g(x)=1$ and $\|g \mid K\|<\epsilon$. Thus $K$ is $H\left(P, \Theta^{n}\right)-$ convex, but since $P$ is polynomial convex, $K$ is also polynomial convex.

6.5. Lemma. Let $S$ be a connected set in $C^{n}$ satisfying: for all $x \in S$ there is a neighborhood $U_{x}$ of $x$ such that $S \cap U_{x}$ is a variety in $U_{x}$ (i.e., $S$ is locally a variety). Suppose $K \subset S$, as a subset of $C^{n}$ is compact and polynomial convex. Then for any $f \in H\left(U \cap S, \Theta^{S}\right), U$ a neighborhood of $K, f \mid K$ is uniformly approximable by polynomials.

Proof. For $x$ in $K$, there is a $U_{x}$ such that $S \cap U_{x}$ is a variety in $U_{x}$. Cover $K$ by finitely many $U_{1}, \cdots, U_{n}$. Let $V_{j}$ open, $\bar{V}_{j}$ compact, contained in $U_{j}$ and $V_{1}, \cdots, V_{n}$ cover $K$. Then $S \cap \bar{V}_{j}$ is compact. Let $K^{\prime}=U_{j=1}^{n} S \cap \bar{V}_{j}$. Then $K^{\prime} \subset S, K^{\prime}$ is compact and int $K^{\prime} \supset K$. By the polynomial convexity, we can find an analytic polyhedron $P=\left\{\left|p_{j}\right|<1 ; j=1, \cdots, k, p_{j}\right.$ polynomials \} such that $K \subset P \cap S \subset$ int $K^{\prime}$. Then $P \cap S$ is closed in $P$ (for $K^{\prime}$ is closed, and $K^{\prime} \cap P=S \cap P$ ). Thus since $P \cap S$ is locally a variety, $P \cap S$ is a variety in $P$. Every $f$ in $H\left(P \cap S, \Theta^{S}\right)$ then is the restriction of $g$ in $H\left(P, \Theta^{n}\right)$ to $P \cap S$ by 6.3 . Since $P$ is polynomial convex, $\left.g\right|_{K}=\left.f\right|_{K}$ is approximable by polynomials, by 6.2 .

6.6. Lemma. $K$ is a compact subset of a Riemann surface. $f_{1}, \cdots, f_{n}$ are holomorphic in a neighborhood $U$ of $K$, and $\omega: U \rightarrow S \subset C^{n}, \omega(r)$ $=\left(f_{1}(r), \cdots, f_{n}(r)\right)$, where $S$ is locally a variety. Suppose $K$ is convex with respect to polynomials in $f_{1}, \cdots, f_{n}$. Then $\omega(K)$ is polynomial convex.

Proof. Let $U \supset \bar{V} \supset V \supset K, V$ a domain, $\bar{V}$ compact. Then $\omega(\bar{V})$ is compact. For $x$ in $\partial \bar{V}$, there is a $p_{x}\left(f_{1}, \cdots, f_{n}\right)$ such that $\left|p_{x}(x)\right|>\left\|p_{x}\right\|_{K}, p_{x}$ a polynomial. Then $\left|p_{x}(\omega(x))\right|>\left\|p_{x}\right\|_{\omega(K)}$. Thus for every $z$ in $\omega(\bar{V})$, there is a polynomial $p_{z}$ such that $\left|p_{z}(z)\right|>\left\|p_{z}\right\|_{\omega(K)}$. By compactness, there are polynomials $p_{1}, \cdots, p_{k}$ such that $\omega(\bar{V}) \supset\left\{\left|p_{j}\right|<1, j=1, \cdots, k\right\} \cap S \supset \omega(K)$. If we add proper multiples of the coordinate functions, we can say that there is an analytic polyhedron $P=\left\{\left|p_{j}\right|<1 ; p_{j}\right.$ polynomials $\}$ such that $K \subset P$ and $P \cap S$ is a variety in $P . P \cap S$ is closed in $P$, for it is the intersection of a closed set, $\omega(\bar{V})$, with $P$. Now $\hat{\omega}\left(H\left(S \cap P, \Theta^{S}\right)\right)$ contains the polynomials in $f_{1}, \cdots, f_{n}$, so by assumption, $\omega(K)$ is $H\left(S \cap P, \Theta^{S}\right)$-convex. Now Lemma 6.4 applies, proving $\omega(K)$ is polynomial convex.

6.7. Theorem. Let $K$ be a compact subset of a Riemann surface $R$, and let $U$ be a neighborhood of $K$. Suppose $A^{0}$ is a subalgebra of $H(U)$ such that: (i) $A^{0}$ is generated by $f_{1}, \cdots, f_{n} \in H(U)$; (ii) $K$ is $A^{0}$-convex; (iii) $\{p \in U$; there is a $q \in U$ such that $p \neq q$ but $f(p)=f(q)$, for all $\left.f \in A^{0}\right\}$ is finite. 
Let $A$ be the uniform closure of $A^{0}$ on $K$. Then there is a variety $V$ in the unit polycylinder of some $C^{n}$ and a map $\phi$ of a neighborhood of $K$ onto $V$ such that

$$
A=\{f \circ \phi ; f \text { in the closure of the polynomials on } \phi(K)\} \text {. }
$$

Proof. We note first that if $K$ is a domain bounded by an analytic arc $\gamma$ and $d f_{1} \neq 0$ on $\gamma$, that (ii) follows from conditions (i) and (iii) by Wermer's Theorem (1.1). On the other hand, the methods of the proof are general for any analytic space, so the theorem still holds if we replace $R$ by any analytic space (of any dimension).

Because of (ii) we can find $p_{1}, \cdots, p_{t} \in A^{0}$ such that $\left\{r \in R ;\left|p_{i}(r)\right| \leqq 1\right.$, $1 \leqq i \leqq t\}=\bar{W}$ is compact on $R$. By multiplication of the $f_{i}$ by a constant we may assume $\left\|f_{i}\right\|_{\bar{W}}<1,1 \leqq i \leqq n$. Then, easily, the map $\phi$ on $W=\{r \in R$; $\left.\left|p_{i}(r)\right|<1, \quad 1 \leqq i \leqq t\right\}$,

$$
\phi(r)=\left(f_{1}(r), \cdots, f_{n}(r), p_{1}(r), \cdots, p_{t}(r)\right),
$$

is a proper holomorphic map of $W$ into $P^{n+t}=\left\{w ;\left|w_{j}\right|<1\right\}$, and $\phi^{-1}(w)$ is finite for all $w$. Thus, by $6.1, \phi(W)=V$ is a subvariety of $P^{n+t}$, and $\phi(K)$ is a compact subset of $\phi(W)$. Now since $K$ is $A^{0}$-convex, it is $H(W)$-convex, and thus $\phi(K)$ is $H(V)$-convex. Thus by $6.4 \phi(K)$ is polynomial convex. It follows that the polynomials are dense in $A(\phi(K))$.

Thus if $f \in A(\phi(K)), f$ is approximable on $\phi(K)$ by polynomials $p_{n}$. Then $p_{n} \circ \phi \mid K$ converges to $f \circ \phi$. But $p_{n} \circ \phi=p_{n}\left(f_{1}, \cdots, f_{n}, p_{1}, \cdots, p_{t}\right)$ is a polynomial in $f_{1}, \cdots, f_{n}, p_{1}, \cdots, p_{t}$, and thus is in $A^{0}$ since $f_{1}, \cdots, f_{n}$, $p_{1}, \cdots, p_{t} \in A^{0}$. Thus $f \circ \phi \in A$. Conversely, if $f \in A$, we have $g_{n} \mid K$ converging to $f$ with $f_{n} \in A^{0}$, in particular $g_{n}=p_{n}\left(f_{1}, \cdots, f_{n}\right)$ where $p_{n}$ is a polynomial. Thus $g_{n}=p_{n} \circ \phi$, so that $f_{n} \in\{f \circ \phi ; f$ a polynomial on $\phi(K)\}$, so $f$ is in the closure of this set, which is $\{f \circ \phi ; f$ in the closure of the polynomials on $\phi(K)\}$. Thus 6.7 is proven.

If $K$ is, in particular, a domain with finitely many piecewise analytic boundary curves, then for any algebra $A^{0}$ satisfying only (ii) and (iii) we can obtain the desired conclusion: that its closure on $K$ can be represented as $A\left(K^{0}\right)$ for $K^{0}$ lying on some analytic space. In this case we can find finitely many functions $f_{1}, \cdots, f_{n}$ satisfying the hypotheses of 6.7 . Thus the algebra $A^{1}$ generated by $f_{1}, \cdots, f_{n}$ on $K$ can be represented as $A\left(K^{1}\right)$, where $K^{1}$ is a compact set on an analytic space $V$. Because only finitely many points can be identified, $K^{1}$ is also a domain bounded by piecewise analytic curves. It is clear that $R$ is the normalization for $V$, and $A$, the closure of $A^{0}$ on $R$ is a subalgebra of $A(K)$ containing $A\left(K^{1}\right)$. Thus $A$ is of finite codimension in $A(K)$, which will guarantee that $A$ is an $A\left(K^{0}\right)$ on some analytic space.

6.8. Corollary. Let $f_{1}, \cdots, f_{n}$ be functions holomorphic in an annulus a about $\{|z|=1\}=\Gamma$. Suppose $f_{1}, \cdots, f_{n}$ separate the points of $\Gamma, d f_{1}$ is never zero on $\Gamma$, and the algebra of $A^{0}=$ uniform closure on $\Gamma$ of polynomials in 
$f_{1}, \cdots, f_{n}$ is not $C(\Gamma)$. Then there is a connected set $S \subset C^{n}$, a closed analytic curve $\gamma$, bounding an open connected subset $\Omega$ of $S$, and a homeomorphism $\phi: \Gamma \leftrightarrow \gamma$ such that: (i) $S$ is locally a variety; (ii) $A^{0}=[f \circ \phi ; f$ in the closure of polynomials on $\Omega \cup \gamma]$.

Proof. Under the assumption Wermer has proven: there exists a Riemann surface $R$, a biholomorphism $\phi^{\prime}: a \leftrightarrow Q^{\prime} \subset R, \phi^{\prime}(\Gamma)=\gamma^{\prime}$, an analytic curve bounding a domain $\Omega^{\prime}$ such that for $A_{1}^{0}=\left[f \circ \phi^{-1} ; f\right.$ is in $\left.A^{0}\right], A_{1}^{0} \subset A\left(\Omega^{\prime} \cup \gamma^{\prime}\right)$ and $A_{1}^{0} \supset \mathscr{g}, \mathcal{I}$ an ideal of $A\left(\Omega^{\prime} \cup \gamma^{\prime}\right)$, with finite hull in $\Omega^{\prime}$.

Let $F_{i}$ be the function in $A\left(\Omega^{\prime} \cup \gamma^{\prime}\right)$ which extends $f_{i} \circ \phi^{-1}$ to all of $\Omega^{\prime} \cup \gamma^{\prime}$. Then $F_{1}, \cdots, F_{n}$ are holomorphic in a neighborhood $U$ of $\Omega^{\prime} \cup \gamma^{\prime}$. Let $P=\left\{\right.$ polynomials in $\left.f_{1}, \cdots, f_{n}\right\}$. Then $P$ is a subalgebra of $H(U)$, and is dense in $A_{1}^{0}$. Then, since $A_{1}^{0}$ contains an ideal with finite hull contained in $\Omega^{\prime}, P$ satisfies (ii) and (iii) of 6.5 and thus we can apply Theorem 6.7 to $A^{0}$.

Appendix. We shall prove a special case of Wermer's Theorem, 1.1. Although this special case in a sense avoids the greatest difficulty in the theorem, that of proving convexity, still it is fairly general and indicates how a proof using the theory of several complex variables might go.

Theorem. Let $M$ be a compact Riemann surface, $K=\Omega \cup_{\gamma}, \Omega$ a domain and $\gamma$ an analytic curve. Let $f_{1}, \cdots, f_{n}$ be meromorphic in $M$ and holomorphic on $K$ such that

$$
N=\left\{p \text { in } M \text {; there is } q \text { in } M \text { such that } f_{j}(p)=f_{j}(q) \text { for all } j=1, \cdots, n\right\}
$$

is finite. Let $A^{0}=$ uniform closure on $K$ of the algebra of polynomials in $f_{1}, \cdots, f_{n}$. Then $A^{0}$ contains an ideal of $A(K)$ of finite codimension.

Proof. We may assume $\left\|f_{j}\right\|_{K}<1$ for $j=1, \cdots, n$. Let $R=\{m$ in $M$; $\left.\left|f_{j}(m)\right|<1\right\}, \bar{R}$ is compact since the $f_{j}$ are meromorphic on $M$. Define $F: R \rightarrow P^{n}=\left\{z ;\left|z_{j}\right|<1,1 \leqq j \leqq n\right\}$,

$$
F(m)=\left(f_{1}(m), \cdots, f_{n}(m)\right) .
$$

(1) $F$ is holomorphic and it is proper. For let $m_{n}$ be in $F^{-1}(A), A$ compact in $P^{n} . \bar{R}$ is compact, so there exists an $m$ in $\bar{R}$ such that $m_{n_{k}} \rightarrow m$. Then $f_{i}(m)$ is a limit point of $\left\{f_{i}\left(m_{n}\right)\right\}$ and since $\left\{f_{i}\left(m_{n}\right)\right\}=\left\{\pi_{i} F\left(m_{n}\right)\right\}$ is contained in a compact subset of the unit disc, we have $\left|f_{i}(m)\right|<1$. Therefore, $F(m)$ $=\left(f_{1}(m), \cdots, f_{n}(m)\right)$ is defined and is in $P^{n}$; since $A$ is compact, $F(m)$ is in $A$. Therefore $m$ in $F^{-1}(A)$; and $F(A)$ is compact.

Now because $N$ is finite, we have that $F^{-1}(x)$ is always finite for $x \in P^{n}$. Thus, by the Abbildungsatz (6.1), $F(R)=S$ is a variety in $P^{n}$.

(2) $(R, F)$ is the normalization for $S$.

Let $s$ be in $S$. Let $F^{-1}(s)=\left\{x_{1}, \cdots, x_{k}\right\}$. Each $x_{i}$ has a neighborhood $U_{i}$ in $S$ such that $\left(U_{i}\right)-\cap N$ is empty, thus $F$ is one-one on $\left(U_{i}\right)^{-}$, and $\left(U_{i}\right)^{-}$is compact. Thus $F: U_{1} \leftrightarrow F\left(U_{i}\right)$ is a homeomorphism. Since $U_{i=1}^{n}\left\{d f_{i}=0\right\}$ is dis- 
crete, we may also assume that except possibly for $x_{i}$, some $d f_{i} \neq 0$ at every point of $U_{i}$. Thus $\left.F\right|_{\left(U_{i}-x_{i}\right)}$ is a holomorphic map of a connected set onto $F\left(U_{i}\right)-s$, so $F\left(U_{i}\right)-s$ is a connected submanifold of $S_{\text {reg }}$ of dimension one, and thus must be an open set contained in one irreducible branch $S_{i}$ of $S$ at $s$. Thus $F\left(U_{i}\right)$ is a neighborhood of $s$ on $S_{i}$. Then $U_{i=1}^{n} F\left(U_{i}\right)$ is a neighborhood of $s$. For suppose $s_{n} \rightarrow s, s_{n}$ is $S$. Let $x_{n}$ be in $R$ such that $F\left(x_{n}\right)=s_{n}$; then by the properness of $F$ there exists an $x$ in $R$ such that $x_{n} \rightarrow x$, so $x$ must be some $x_{i}$, since $F(x)=s$. But then the $x_{n}$ from some $n^{0}$ on must be in $U_{i}$, so $s_{n}$ in $F\left(U_{i}\right)$. Thus $\bigcup_{i=1}^{n} F\left(U_{i}\right)$ is a neighborhood of $s$ on $S$.

Let $s$ be in $S_{\text {sing. }}$. The branches of $S$ at $s$ are just the $F\left(U_{i}\right)$ described above, then $s$ is an identification point if and only if $F^{-1}(s)$ is more than one point. If $F^{-1}(s)=x$, then we must have here $d f_{j}(x)=0,1 \leqq j \leqq n$, for if not then $F$ is biholomorphic in a neighborhood of $x$, which would make $S$ a manifold at $s$. Conversely, if $s$ is in $S_{\text {reg }}$, then $d z_{j}(s) \neq 0$ for some $j$, i.e., $z_{j}$ is one-one in a neighborhood of $S$, implying $d f_{j}(x) \neq 0$. Thus $F^{-1}\left(S_{\text {sing }}\right)$ $=N \cup\left\{d f_{j}=0,1 \leqq j \leqq n\right\}$, which is a finite set (since $\bar{R}$ is compact). Now, for $x$ not in $F^{-1}\left(S_{\text {sing }}\right)$ we have that some $d f_{j} \neq 0$, and $F$ is a homeomorphism in a neighborhood of $x$ onto a neighborhood of $F(x)$; it follows that $F$ is biholomorphic in a neighborhood of $x$. Thus $\left.F\right|_{R-F^{-1}\left(\Omega_{\mathrm{Atg}}\right)}$ is biholomorphic. Thus $(R, F)$ is the normal model for $S$.

It is easy to see that $K$, since it consists of a domain bounded by one analytic curve, can be written as the decreasing intersection of domains simply connected with respect to $R$. Then, by $4.1, K$ is $H(R)$-convex. Then $K^{0}=F(K)$ is also $H(S)$-convex, as in Theorem 4.4, for in this case $K=\tilde{K}^{0}$. Then by $6.4, K^{0}$ is polynomial convex.

Now, as in $\S 5,\left\{f \circ F ; f\right.$ in $\left.A\left(K^{0}\right)\right\}$ contains an ideal $I$ of $A(K)$ of finite codimension. If $f$ is in $H\left(U, \Theta^{S}\right), U$ a neighborhood on $S$ of $K$, then by 6.3,

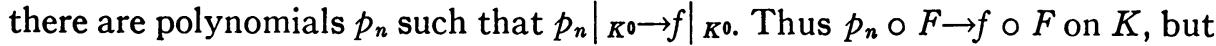
$p_{n} \circ F=p_{n}\left(f_{1}, \cdots, f_{n}\right)$ is in $A^{0}$. Thus $f \circ F$ is in $A^{0}$, and finally $I \subset A^{0}$ (in fact, by 6.7, $\left\{f \circ F ; f\right.$ in $\left.\left.A(K)^{0}\right)\right\}=A^{0}$ ).

COROLLARY. If in addition we assume that $f_{1}, \cdots, f_{n}$ separate the points of $K$ and for all $m$ in $K$ there is an $f_{j}$ such that $d f_{j}(m) \neq 0$, then $A^{0}=A(K)$.

Proof. In this case there is a neighborhood $U$ of $K$ such that $\left.F\right|_{U}$ is biholomorphic. Then $\hat{F}\left(H\left(F(U), \Theta^{S}\right)\right)=H\left(U, \Theta^{R}\right)$. Since $f$ in the former is approximable on $K$ by polynomials in $f_{1}, \cdots, f_{n}$, then $A^{0}$ is dense in $H\left(U, \Theta^{R}\right)$. The latter is dense in $A(K)$; thus $A^{0}=A(K)$.

\section{BiBLIOGRAPHY}

1. R. Arens, The closed maximal ideals of algebras of functions holomorphic on a Riemann surface, Rend. Circ. Mat. Palermo vol. 7 (1958) pp. 1-16.

2. W. L. Baily, Several complex variables, University of Chicago notes, 1957.

3. H. Behnke, Généralisation du théorème de Runge pour des fonctions multiformes de variables complexes, Colloque sur les fonctions de plusieurs variables, Paris, 1953. 
4. E. Bishop, Subalgebras of functions on a Riemann surface, Pacific J. Math. vol. 8 (1958) pp. 29-50.

5. - Seminaire H. Cartan de l'Ecole Normale Superieure, 1951-1952 (chapters VIIXX, Math. Dept. of M. I. T.).

6. H. Grauert and R. Remmert, Komplexe Raume, Math. Ann. vol. 136 (1958) pp. 245-318.

7. K. Hoffman and I. M. Singer, Maximal algebras of continuous functions, Acta Math. vol. 103 (1960) pp. 217-241.

8. K. Oka, Sur les fonctions analytiques de plusieurs variables. VII, Lemme fondamental, J. Math. Soc. Japan vol. 3 (1951) pp. 204-214, 259-278.

9. R. Remmert, Holomorphe und meromorphe Abbildungen komplexer Räume, Math. Ann. vol. 133 (1957) pp. 328-370.

10. H. Royden, On a theorem of Wermer's, Stanford University Applied Math. and Stat. Lab. Technical Report no. 9, 1959.

11. J. Wermer, Subalgebras of the algebra of all complex-valued continuous functions on the circle, Amer. J. Math. vol. 78 (1956) pp. 225-242.

12. - Rings of analytic functions, Ann. of Math. vol. 67 (1958) pp. 45-71.

13. - The hull of a curve in $C^{n}$, Ann. of Math. vol. 68 (1958) pp. 550-561.

Massachusetts Institute of Technology,

Cambridge, Massachusetts

PrinCETON UNIVERSITY,

Princeton, New Jersey 